\title{
A method of continuous nonlinear gamma correction
}

\author{
Xue-Wen Zhang \\ School of art, Gansu Industry Polytechnic College, \\ Tianshui, Gansu, China \\ Hao-Dong Shen', Xiao-Long Xu and Zhuo Zhang \\ College of Internet of Things Engineering, Hohai University, \\ Changzhou, Jiangsu, China \\ E-mail:shenhd_1991@163.com
}

\begin{abstract}
Uneven illumination in image acquisition process often have a serious impact on image processing. For the deficiency of traditional adaptive Gamma correction in the process of light correction, we present a method of continuous nonlinear Gamma correction. The proposed method has effective compensation for the highlights and shadows at same time, and a faster processing speed than other methods. Experiments show that the method can effectively solve the problem of the non-uniform illumination effects, and improve the quality of the image.
\end{abstract}

Key words: Uneven illumination; Gamma correction; Continuous nonlinear.

\section{Introduction}

In image acquisition, object occlusion, shooting angle, light intensity and other reasons often lead to highlight and shadow areas in the image. And the large amount of missing details in the highlight and shadow areas often brings great inconvenience to the follow - up process. Therefore, it is an important research direction in image processing to make illumination compensation on image and enhance the details of the highlight and shadow regions in the preprocessing [1-5]. At present, there is a wide range of research and application of adaptive Gamma correction to compensate the illumination [6-14].

The earliest adaptive Gamma correction method is proposed in [6], it divides gray-scale value of image into three parts: the highlights, the transition and the shadow, and then establishes the nonlinear relationship between gray-scale value and Gamma value respectively. It overcomes the shortcomings of traditional Gamma correction that can't compensate the illumination on the highlight and shadows at the same time. However, the value of Gamma in this method is not well adapted to the change of illumination and the image distort after correction. In [7] and [8], an improved method is proposed to overcome the shortcomings of [6]. In [7], a nonlinear function is added. In [6], the function is 
revised, which improves the Gamma's adaptability to illumination variations and avoid the distortion of the image after correction. In [8], the second nonlinear function of [7] is replaced, and a parameter is introduced. By changing the parameter values, the highlight, transition and shadow regions of different images are divided reasonably. But it fails to solve the problem of how to adaptively select this parameter.

Recent years, there are three main methods: Local Gamma Correction Method (LGC) [9], Adaptive Gamma Correction with Weighting Distribution (AGCWD) [10], and Adaptive Gamma Correction with Color Preserving Framework (AGCWPF) [11]. The LGC method is similar to the method proposed in [6], it divides the image pixels into three parts: highlight, transition and shadow. And each part adopts different Gamma values, although it meets the requirement of illumination compensation for highlight and shadow regions at the same time, the Gamma value of this method also can't adapt to the change of illumination. AGCWD establishes the relationship between Gamma value and gray-scale value distribution. Based on AGCWD, AGCCPF introduces histogram equalization. The original image histogram is linearly superimposed with the histogram after equalization as the new gray distribution. Both of the methods improve the contrast of the image, but increase the gray-scale value of all the pixels at the same time, and can't compensate the highlight regions of the image effectively.

For the shortcomings of the above methods, a continuous nonlinear Gamma correction method is presented in this paper, through the tangent function, it establish the nonlinear relationship between gray-scale value and Gamma value. This method can make the Gamma value adapt well to the change of illumination, and avoid the distortion of the image after correction, and then it only use one nonlinear function which reduces the complexity of the algorithm.

\section{Adaptive Gamma Correction}

Gamma correction is a commonly used method of gray-scale transformation, for gray-scale image of $0 \sim 255$, its basic form is shown in equation (1). Where $x$ is the gray-scale value before Gamma correction, $y$ is the gray-scale value after Gamma correction.

$$
y=255 \times\left(\frac{x}{255}\right)^{\gamma}
$$

For different values of $\gamma$, the relationship of $x$ and $y$ is shown in Figure 1, it can be found that,

1) When $\gamma=1$, the corrected gray-scale value is consistent with the gray-scale value before correction; 
2) When $\gamma<1$, the gray-scale value of the corrected image is higher than that before correction, and it increases obviously in the low gray-scale value regions; 3) When $\gamma>1$, the gray-scale value after correction is smaller than that before correction, and it decreases obviously in high gray-scale value regions.

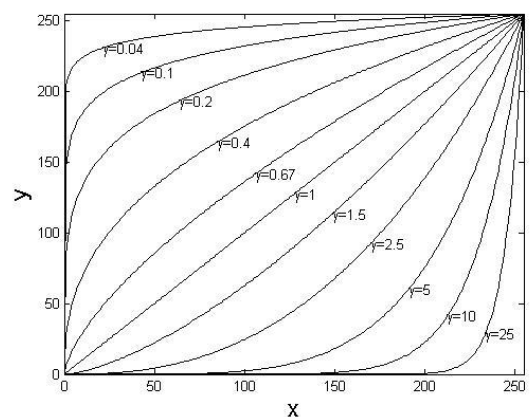

Fig. 1. The relationship between $y$ and $x$ with different $\gamma$.

If you apply a fixed Gamma value to all the pixels in the image for correction, the whole image will darken or lighten. However, most of the images are non-uniform illumination, it is widespread that some areas are too bright, some are dark, the fixed Gamma value can't be used to effectively compensate illumination for the shadow and highlight region at the same time.

Therefore, construct a reasonable function to establish the relationship between Gamma value and gray-scale value, that Gamma value is less than 1 in the low gray-scale value regions and higher than 1 in the high gray-scale value regions, can increase the pixel gray-scale value of the image shadow regions, decrease the pixel gray-scale value of highlight regions, therefore, the shadow and the highlight region can be compensated illumination effectively at the same time.

\section{Continuous Nonlinear Gamma Correction}

Different with the methods in [6] [7] that simply divide gray-scale value into three parts: the highlight, the transition and the shadow, the gray-scale value from low to high is looked as continuous transition from shadow to highlight in this paper. We consider constructing a continuous nonlinear function to establish the relationship between the gray-scale value and the Gamma value, which meet the requirement that the shadow and highlight regions deviated from the median gray-scale value compensated larger illumination and that near the median gray-scale value compensated smaller illumination. That is, the relation curve of Gamma value and gray-scale value has a higher slope at both ends and a smaller slope in the middle position. Therefore, based on the tangent function, the 
nonlinear relationship between Gamma value and gray-scale value as described in formula (2) is established in this paper.

$$
\gamma(x)=1+c \times \tan \left(\frac{x-128}{s} \pi\right)
$$

Where $x$ is the gray-scale value before correction, $s$ stands for the range of the tangent function angle value, and $c$ stands for the range of the gamma value $\varphi(x)$ is defined as follows.

$$
\varphi(x)=\frac{x-128}{s} \pi
$$

Taking into account that $\varphi(x) \in(-\pi / 2, \pi / 2)$, that is $[(0-128) \pi / s,(255-128) \pi / s]$ $\subset(-\pi / 2, \pi / 2)$, so $s$ need to meet the following requirement, $s \in(256,+\infty)$.when the domain of $\gamma(x)$ meet the requirement that $\gamma(x) \in[1-a, 1+a]$, the relationship of $c$ and $s$ is defined as equation (4), where $a<1$.

$$
c=a \times \frac{1}{\tan \left(\frac{128}{s} \pi\right)}
$$

When the range of $\gamma(x)$ is defined as $\gamma(x) \in[0.5,1.5]$, for the different value of $s$, the relationship of Gamma value and gray-scale value is shown in Figure 2.

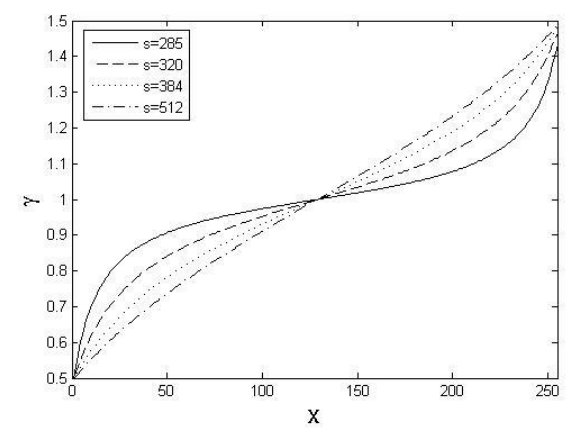

Fig.2. The relationship between Gamma and Gray with different $s$.

It can be found that, for any value of $s$, from the two ends to the middle, the change of Gamma value with the gray-scale value is gradually slowdown from the steep, and the curve is a little steep overall when value $s$ is smaller, but it gradually become smoothly with the increase of value $s$, when $s=512$, the curve is close to a straight line.

When the range of $\gamma(x)$ is defined as $\gamma(x) \in[0.75,1.25], \gamma(x) \in[0.5,1.5]$ and $\gamma(x) \in[0,2]$ respectively, for the different value of $s$, the relationship of Gray-scale value before and after correction is shown in Figure 3. It can be 
found that, with the expansion of the range of $\gamma(x)$, the correction of gray-scale value are more obvious for any value of $s$. But when $\gamma(x)$ out of range, for some $s$, the correction value of low-gray-scale regions deviate from the normal range apparently, and the original information of the image has been lost (shown in figure 3(c) and 3(d)).

Therefore, select the appropriate value of $s$ and $a$ experimentally, control the value of $\varphi(x)$ and $\gamma(x)$ within a reasonable range, so that the algorithm proposed in this paper is effective in compensating illumination as well as preserving the effective information of the original image.

(a)

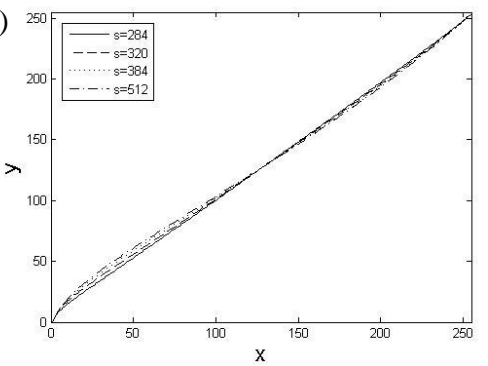

(c)

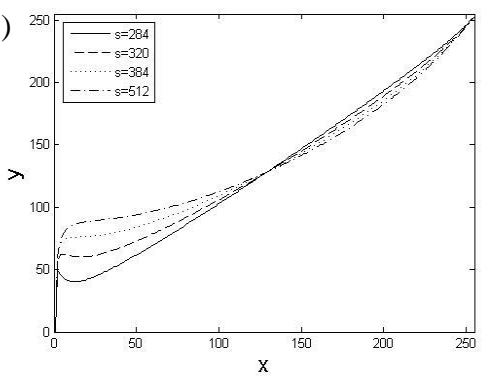

(b)

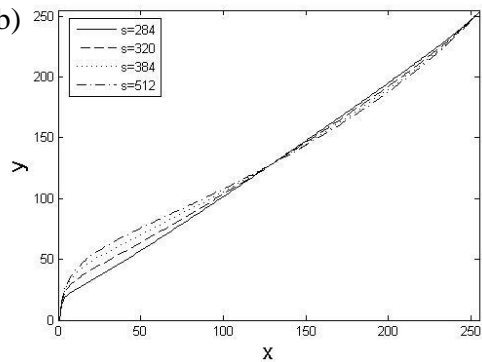

(d)

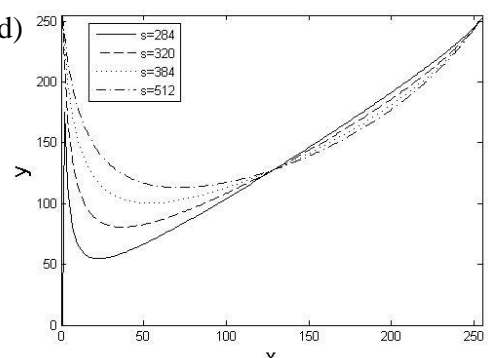

Fig. 3. The Gray relationship between before and after with different values of $\gamma(x)$ (a) $\gamma(x) \in[0.75,1.25]$;(b) $\gamma(x) \in[0.5,1.5]$;(c) $\gamma(x) \in[0.25,1.75]$;(d) $\gamma(x) \in[0,2]$

\section{Experiment Results}

This paper select the appropriate value of $s$ and $a$ based on information entropy[15, 16], a common indicator of the image clarity, and then make a comparison with other methods. The information entropy is defined as Equation (5), where $p_{i}$ represents the ratio of the pixels with the gray-scale value of $i$ to the total pixels.

$$
E=-\sum_{i=0}^{L-1} p_{i} \log p_{i}
$$

In this experiment, the experimental data which has highlight and shadow is selected in the AFW (The Annotated Faces in the Wild Test Set) data set. 
Visual comparison of different algorithms: our algorithm (use $s=365$, $a=0.4$ as an example), literature[6], literature[7], literature[8], LGC, AGCWD, AGCCPF , on a highlight image is shown as figure 4. It can be found that, The method proposed in this paper and literature [6] [7] can effectively reduce the gray-scale value of the highlight regions, and make the right face of the character relatively clear, the effect of the method in [8] is not very obvious, by contrast, the method of LGCTT,AGCWD and AGCCPF increase the gray-scale value of the highlight regions so that causing the image over exposure.

(a)

(e)

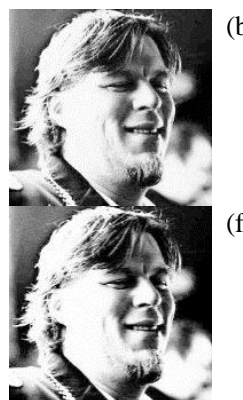

b)

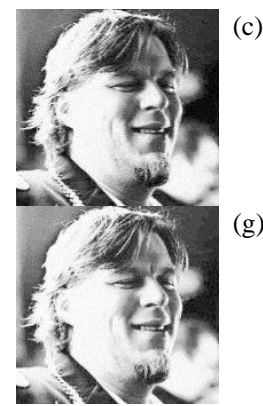

(c)

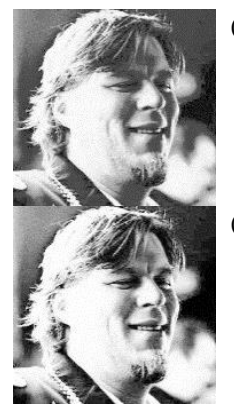

(d)

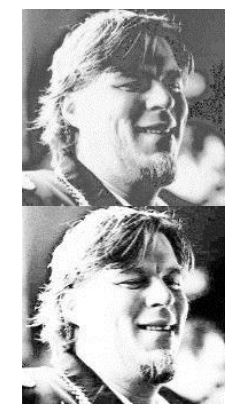

Fig. 4. Several kinds of illumination compensation method for specular image processing effect (a)original; (b)algorithm proposed in this paper; (c)literature[6]; (d)literature[7]; (e)literature[8]; (f)LGC;(g)AGCWD;(h)AGCCPF

(a)

(e)

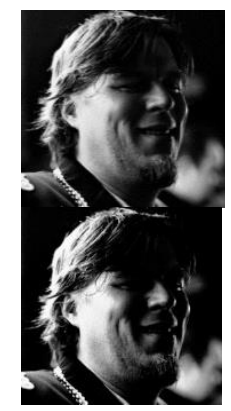

(b)

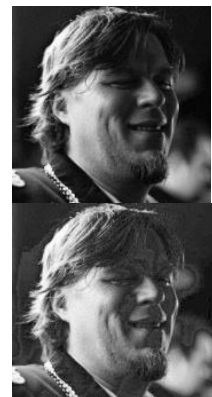

(c)

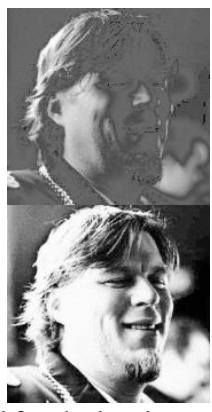

(d)

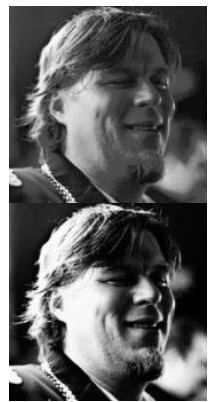

Fig. 5. Several kinds of illumination compensation method for shadow image processing effect (a)origina; (b)algorithm proposed in this paper; (c)literature[6]; (d)literature[7]; (e)literature[8]; (f)LGC;(g)AGCWD;(h)AGCCPF

Visual comparison of different algorithms: our algorithm (use $s=365$, $a=0.4$ as an example), literature [6], literature [7], literature [8], LGC, AGCWD, AGCCPF, on a shadow image is shown as figure 5. It can be found that, The method proposed in this paper and literature [7] can effectively increase the gray-scale value of the shadow regions, and make the left face of the character relatively clear, the effect of LGC and the method in [6] is not very obvious, and the method of AGCWD and AGCCPF increase the gray-scale value of the shadow regions but causing overexposure in parts of the image. 
Table 1. The entropy of the processed specular images by several illumination compensation method

\begin{tabular}{lllllllll}
\hline & Image1 & Image2 & Image3 & Image4 & Image5 & Image6 & Image7 & Image8 \\
\hline$s=365, a=0.4$ & 5.1085 & 5.2042 & 5.0598 & 5.1290 & 5.1495 & 5.1403 & 5.1512 & 5.0225 \\
$s=384, a=0.4$ & 5.1096 & 5.2034 & 5.0606 & 5.1268 & 5.1473 & 5.1350 & 5.1497 & 5.0209 \\
$s=427, a=0.4$ & 5.1118 & 5.2052 & 5.0566 & 5.1282 & 5.1495 & 5.1390 & 5.1521 & 5.0191 \\
literature [6] & 4.9998 & 5.0227 & 4.8514 & 4.9314 & 4.9738 & 5.0373 & 4.8665 & 4.5740 \\
literature [7] & 4.9600 & 4.9868 & 4.8640 & 4.9719 & 4.9899 & 4.8724 & 4.9225 & 4.7458 \\
literature [8] & 4.8731 & 5.1040 & 4.7870 & 4.8918 & 4.8763 & 5.0031 & 4.9696 & 4.7661 \\
LGCTT & 4.8442 & 4.9893 & 4.8350 & 4.9091 & 4.9626 & 5.0340 & 4.9547 & 4.8738 \\
AGCWD & 4.8605 & 4.9930 & 4.7760 & 4.8542 & 4.8857 & 5.0753 & 4.8941 & 4.7636 \\
AGCCPF & 4.5797 & 4.7836 & 4.4974 & 4.5806 & 4.6362 & 4.8764 & 4.6675 & 4.5125 \\
\hline
\end{tabular}

The information entropy comparison of different algorithms: our algorithm, literature [6], literature [7], literature [8], LGC, AGCWD, AGCCPF, on a highlight image is shown as table 1. It can be found that the information entropy of the image processed by our method is higher than other methods, and in most cases, it has a maximum information entropy when $s=427, a=0.4$.

Table 2. The entropy of the processed shadow images by several illumination compensation method

\begin{tabular}{lllllllll}
\hline & Image1 & Image2 & Image3 & Image4 & Image5 & Image6 & Image7 & Image8 \\
\hline$s=365, a=0.4$ & 5.1456 & 4.7351 & 5.0022 & 4.9934 & 5.0759 & 4.7345 & 4.8175 & 5.182 \\
$s=384, a=0.4$ & 5.1475 & 4.7033 & 4.9903 & 4.9813 & 5.0732 & 4.7188 & 4.7927 & 5.1795 \\
$s=427, a=0.4$ & 5.1607 & 4.7364 & 4.9878 & 5.0106 & 5.0924 & 4.7312 & 4.8212 & 5.1796 \\
literature [6] & 4.7455 & 4.5034 & 4.9844 & 4.6412 & 4.7072 & 4.5635 & 4.7272 & 4.9675 \\
literature [7] & 4.4669 & 3.5297 & 3.9979 & 3.9678 & 4.1252 & 3.7706 & 3.7807 & 4.3727 \\
literature [8] & 4.8356 & 3.8589 & 4.0331 & 4.3947 & 4.6327 & 4.0539 & 4.0608 & 4.5852 \\
LGCTT & 5.2043 & 4.6325 & 5.025 & 4.9603 & 5.1516 & 4.6535 & 4.7136 & 5.201 \\
AGCWD & 5.3842 & 5.3395 & 5.3229 & 5.3942 & 5.3897 & 4.9826 & 5.2155 & 5.2767 \\
AGCCPF & 5.3487 & 5.1345 & 5.1766 & 5.2707 & 5.3556 & 4.8162 & 5.0306 & 5.247 \\
\hline
\end{tabular}

The information entropy comparison of different algorithms: our algorithm, literature [6], literature [7], literature [8], LGC, AGCWD, AGCCPF, on a shadow image is shown as table 2. It can be found that the information entropy of the image processed by our method is higher than the methods in [6][7][8], for a few images, it is slightly lower than LGCTT, AGCWD and AGCCPF method, for most image, it is close to LGCTT, AGCWD and AGCCPF method. And in most cases, the method in this paper has a maximum information entropy when $s=427, a=0.4$.

In summary, the algorithm proposed in this paper is superior to the method in [6], [7], [8], LGC, AGCWD and AGCCPF in illumination compensation for highlight images; for shadow images, this algorithm is superior to the method in 
[6], [7], [8], but close to the method of LGCTT, AGCWD and AGCCPF, and it has the best effect when $s=427, a=0.4$

\section{Conclusions}

According to the different compensation requirements of the no uniform illumination image in the highlights and shadows, combining the method of [6] and [7], this paper proposes a continuous nonlinear Gamma correction method regarding the gray-scale value from low to high as the continuous transition from shadow to highlight. The theoretical and experimental results show that:

(1) The algorithm proposed in this paper can adapt well to the change of illumination without distortion.

(2) The algorithm proposed in this paper has better compensation effect for the highlight and shadow regions.

But it is limited to improve the image clarity which need to further improve later.

\section{Acknowledge}

This work was partially supported by National Natural Science Foundation of China No.61671202, No.61573128, No.61273170 and No.41301448; the Fundamental Research Funds for the Central Universities No.2015B25214. Work partially supported by grant $2-4570.5$ of the Swiss National Science Foundation.

\section{Reference}

1. Yun, S., J.H. Kim and S. Kim, Image enhancement using a fusion framework of histogram equalization and Laplacian pyramid, IEEE Transactions on Consumer Electronics56, 2763(2010).

2. Nercessian, S.C., K.A. Panetta and S.S. Agaian, Non-Linear Direct Multi-Scale Image Enhancement Based on the Luminance and Contrast Masking Characteristics of the Human Visual System, IEEE Transactions on Image Processing22, 3549(2013).

3. Bai, X., F. Zhou and B. Xue, Image enhancement using multi scale image features extracted by top-hat transform, Optics \& Laser Technology44, 328(2012).

4. Du, S. and R.K. Ward, Adaptive Region-Based Image Enhancement Method for Robust Face Recognition Under Variable Illumination Conditions, IEEE Transactions on Circuits and Systems for Video Technology20, 1165(2010).

5. Singh, K. and R. Kapoor, Image enhancement using Exposure based Sub Image Histogram Equalization, Pattern Recognition Letters36, 10(2014). 
6. J. Yang, Z. Fu, T. Tan and W. Hu, A novel algorithm for content-based image recognition and filtering, Journal of China Institute of Communications, 93(2004).

7. J. Yang, Y. Shi and X. Xiong, Improved Gamma Correction Method in Weakening Illumination, Journal of Civil Aviation University of China, 39(2006).

8. P. Liu, J. Wang and Z. Yin, Study on Illumination Compensation Method for Images, System Engineering and Electronic30, 1343(2008).

9. Shi, J. and Y. Cai. A novel image enhancement method using local Gamma correction with three-level thresholding, (ITAIC), (Chongqing, China, 2011).

10. Huang, S., F. Cheng and Y. Chiu, Efficient Contrast Enhancement Using Adaptive Gamma Correction With Weighting Distribution, IEEE Transactions on Image Processing22, 1032(2013).

11. Gupta, B. and M. Tiwari, Minimum mean brightness error contrast enhancement of color images using adaptive gamma correction with color preserving framework, Optik - International Journal for Light and Electron Optics127, 1671(2016).

12. Chiu, Y., F. Cheng and S. Huang. Efficient contrast enhancement using adaptive gamma correction and cumulative intensity distribution, In (SMC), (Kyoto, Japan, 2011).

13. Jiao, Z. and B. Xu, Color image illumination compensation based on HSV transform and homomorphic filtering, Computer Engineering and Applications46, 142(2010).

14. Lee, S., et al., A space-variant luminance map based color image enhancement, IEEE Transactions on Consumer Electronics56, 2636(2010).

15. H. Wang, W. Zhong, J. Wang and D. Xia, Research of Measurement for Digital Image Definition, Journal of Image and Graphics9, 828(2004).

16. W. Zheng and Y. Dai, Adaptive logarithm for facial illumination compensation based on homomorphic gomomorphic module, Journal of Image and Graphics16, 1429(2011). 